\title{
RESEARCH OF THE RESONANCE PROPERTIES OF HELMHOLTZ RESONATORS
}

\author{
Yuliia Kopytko ${ }^{1}$, Vitaliy Zaets ${ }^{2}$, Sergey Naida $^{3}$, Vitaliy Didkovskyi ${ }^{4}$, Anastasiia Damarad ${ }^{5}$ \\ ${ }^{1} 1$ Department of Acoustic and Multimedia Electronic Systems, National Technical University of Ukraine "Igor Sikorsky Kyiv Polytechnic \\ Institute", Kyiv, Ukraine \\ ${ }^{4}$ Department of Acoustic and Multimedia Electronic Systems, National Technical University of Ukraine "Igor Sikorsky Kyiv Polytechnic \\ Institute", Kyiv, Ukraine \\ ${ }^{5}$ Department of Acoustic and Multimedia Electronic Systems, National Technical University of Ukraine "Igor Sikorsky Kyiv Polytechnic \\ Institute", Kyiv, Ukraine

ARTICLE INFO

Article history:

Received date 23.06.2020

Accepted date 22.07.2020

Published date 31.08.2020

Section:

Machine building and metal processing

D O I

$10.21303 / 2313-8416.2020 .001389$

\section{KEYWORDS}

Helmholtz resonator

resonance frequency

resonator in an acoustically rigid shield

Helmholtz resonator modeling

natural frequency

\section{ABSTRACT}

The object of research. Process of oscillation of the Helmholtz resonators.

Investigated problem. Differences between some formulas for the calculation of the resonant frequency of the Helmholtz resonator and the most accurate of them were established. The effect of acoustic design on the Helmholtz resonator frequency value and influence of the attached air mass between the neck of the Helmholtz resonator and free field were investigated.

The main scientific results. As a result of a numerical experiment, analytical ratios were obtained that allow obtaining the most accurate results. One of them is the most optimal for calculating the resonator resonant frequency in a free field, and in this case, less than $1 \%$ inaccuracy level can be achieved, given that $r / R<0.25$. Other one allows to achieve the same low inaccuracy level for a resonator located in an acoustically rigid shield, given that $r / R<0.25$. Research has shown that the location of the resonator in an acoustically rigid shield leads to significant changes in natural resonance frequency value.

The area of practical use of the research results. The detailed research of previously unexplored properties of resonators will make it possible to improve the algorithms for the development of metamaterials, to discover additional parameters with which it is possible to control the characteristics of the metamaterial.

Scope of the innovative technological product. Such resonators are used as the basic elements in metamaterials, as structural elements of the sound-absorbing panels, in acoustic mufflers.

(C) The Author(s) 2020. This is an open access article under the CC BY license http://creativecommons.org/licenses/by/4.0).

\section{Introduction}

\section{1. The object of research}

The influence of acoustic design on the natural resonance frequency value of the investigated Helmholtz resonators was estimated. The accuracy of calculation of the natural resonance frequency of such resonators for some calculation formulas was estimated.

\section{2. Problem description}

In recent years, the research of Helmholtz resonators properties has become an increasingly popular topic for scientific experiments and theoretical research. The increased relevance of this 
direction of research is associated with the active use of such resonators in the structure of metamaterials intended for focusing (example, in [1, 2]), controlling the direction of sound (example, in [3]) or to achieve an extraordinary sound loss (example, in [4, 5]), as basic elements, cells. Thus, a detailed research of previously unexplored properties of resonators will make it possible to improve the algorithms for the development of metamaterials, to discover additional parameters with which it is possible to control the characteristics of the metamaterial. The influence of Helmholtz resonator geometry on its characteristics was researched in many works (example, [6, 7]).

This work proposes a research of the effect of acoustic design on the Helmholtz resonator frequency value. In practice, it was found that the value of the Helmholtz resonator resonant frequency strongly depends on its acoustic design, but practically no researches related to this issue have been carried out. The Helmholtz resonators resonant frequencies for free resonator cases and for its attachment in three different plate's cases were investigated in [8]. The resonators were attached to the plate with neck parts, the latter remained were open. As a result of the experiment, it was found that the resonant frequencies of the resonator in the plates will be lower than the frequency of resonator natural vibrations. In work [9], researches of resonators enclosed in a melamine casing were carried out. The resonant frequency of the construction, which was matched to the Helmholtz resonator natural frequency (in this article), was obtained. Accordingly, the assessment of the influence of presence of acoustic design on the resonator resonant frequency was not carried out in the article.

\section{3. Suggested solution to the problem}

Assessing the effect of acoustic design on the frequency characteristics of an individual resonator at the early stages of metamaterial development can provide an additional way of tuning the material to operate at a given frequency by selecting the acoustic design parameters.

The aim of this research is to research the accuracy of resonant frequency calculation formulas of the Helmholtz resonator and to research the effect of acoustic design on the natural resonance frequency value.

\section{Materials and methods}

Carrying out full-scale experiments in the study of Helmholtz resonators (especially small sizes) are associated with objective difficulties, specifically, the size of the microphones. By placing a microphone in a cavity or throat, let's make an additional change in air volume and air mass, which significantly affects the measurement results. The way out of this situation is to carry out computer modeling. As shown in work [10], computer simulation of resonators in the Comsol Multiphisics environment gave fairly accurate results for large resonators, where the effect of microphones sizes was minimal. Therefore, in this study, it was proposed to construct a computer model of a classical resonator in the form of a cylindrical neck, connected to a spherical volume (Fig. 1). A computer model of such resonators in Comsol Multiphisics environment makes it possible to evaluate the effect of the resonator size on the value of the resonant frequency.

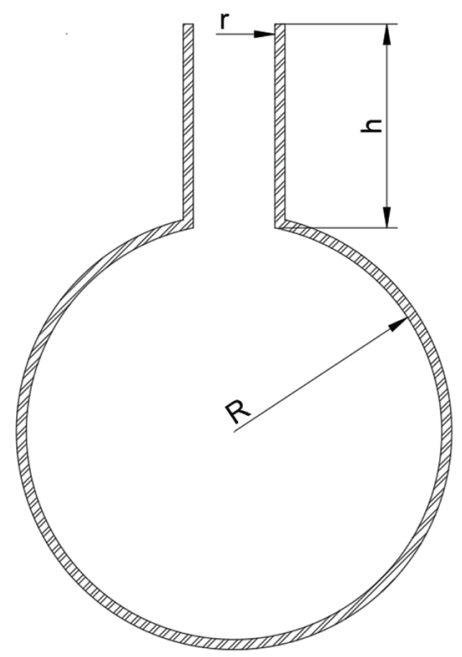

Fig. 1. General view of the resonator 
The questions of resonators resonant frequency calculation were studied 150 years ago, and the most famous formula was derived by Helmholtz:

$$
f=\frac{c}{2 \pi} \sqrt{\frac{S}{V \cdot h}},
$$

where $c=343 \mathrm{~m} / \mathrm{s}-$ speed of sound in air; $S=2 \pi r-$ neck cross-sectional area; $V=\frac{4}{3} \pi R^{3}-$ spherical cavity volume; $h$ - neck length.

Later this formula was refined by many scientists who tried to take into account the added air mass in the "inner" area - between the neck and the spherical volume, and the "outer" area -the attached air mass between the neck and free field. One of the versions for this formula (2) [11]:

$$
f=\frac{c}{2 \pi} \sqrt{\frac{S}{V \cdot(h+1,7 \cdot r)}} .
$$

However, both of these formulas (1) and (2) did not take into account the condition of surrounding field, specifically, whether the resonator is located in an infinite field or is fixed in acoustically rigid shield, as show in Fig. 2.

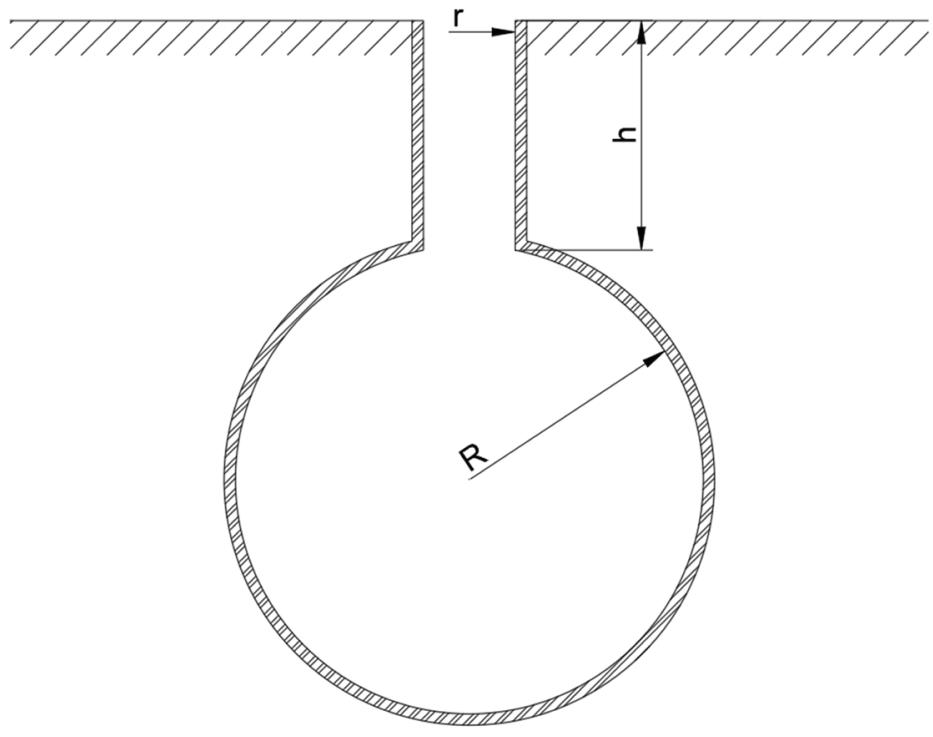

Fig. 2. Resonator in an acoustically rigid shield

\section{Results}

To obtain the results and further analyze them, a numerical experiment was carried out. The resonant frequency of the resonator at $f=100 \mathrm{~Hz}$ and the constant radius of the resonator neck $r=0.02 \mathrm{~m}$ were taken as a basis. The length of the neck $\mathrm{h}$ and the radius of the spherical volume were the variable parameters. Results of calculating the height (p. 3) and the radius of the spherical volume (p. 4) are presented in Table 1.

The same table shows the results of calculating the resonant frequency (p. 5) obtained using a computer model, taking into account the calculated geometric parameters of the resonator.

As can be seen from the results of the calculation, the value of the resonant frequency (p. 1) calculated by formula (1) does not correspond to the obtained values of computer simulation (p. 5). This fact is not new, however, the results of calculating the resonant frequency (p. 6), carried out using the improved formula (2), also showed a significant inaccuracy (p. 7). When constructing acoustic metamaterials, such inaccuracy in the estimation of the resonance frequency is unsatisfactory. 
To reduce the inaccuracy in calculating the resonant frequency, formula (3) was proposed, which, in contrast to the previous formulas, provides a significantly smaller calculation inaccu$\operatorname{racy}(\mathrm{p} .11)$.

$$
f=\frac{c}{2 \pi} \sqrt{\frac{(V-\Delta V) \cdot\left(h+\frac{4}{3\left(\pi-\arcsin \left(\frac{R-r}{R+h}\right)\right)} r+\frac{4}{3 \cdot \arccos \left(\frac{r}{R}\right)} r\right)}{(2)}},
$$

$\Delta V=\frac{1}{3} \pi\left[3 R\left(R-\sqrt{\left(R^{2}-r^{2}\right)}\right)^{2}-\left(R-\sqrt{\left(R^{2}-r^{2}\right)}\right)^{3}\right]-$ the volume of a sphere segment cut out by a cylindrical neck.

Table 1

Results of a numerical experiment for a resonator with $100 \mathrm{~Hz}$ frequency

\begin{tabular}{|c|c|c|c|c|c|c|c|c|c|c|}
\hline No. & Parameter & \multicolumn{9}{|c|}{ Parameter value } \\
\hline \multicolumn{11}{|c|}{ Without acoustic design } \\
\hline 1 & $f_{1}, \mathrm{~Hz}$ & & & & & 100 & & & & \\
\hline 2 & $r, \mathrm{~m}$ & & & & & 0.02 & & & & \\
\hline 3 & $h, \mathrm{~m}$ & 0.5 & 0.2 & 0.1 & 0.05 & 0.02 & 0.01 & 0.005 & 0.002 & 0.001 \\
\hline 4 & $R, \mathrm{~m}$ & 0.0564 & 0.0765 & 0.0963 & 0.1214 & 0.1647 & 0.2075 & 0.2615 & 0.3549 & 0.4471 \\
\hline 5 & $f_{C}, \mathrm{~Hz}$ & 86.6 & 91.7 & 87.8 & 79.5 & 63.4 & 49.8 & 37.4 & 24.6 & 17.7 \\
\hline 6 & $f_{2}, \mathrm{~Hz}$ & 96.8 & 92.5 & 86.4 & 77.2 & 60.9 & 47.7 & 35.8 & 23.6 & 16.9 \\
\hline 7 & $\delta, \%$ & -11.78 & -0.87 & 1.59 & 2.89 & 3.94 & 4.22 & 4.28 & 4.07 & 4.52 \\
\hline 8 & $f_{3}, \mathrm{~Hz}$ & 97.1 & 93.4 & 87.9 & 79.3 & 63.1 & 49.5 & 37.2 & 24.4 & 17.5 \\
\hline 9 & $\delta, \%$ & -12.10 & -1.81 & -0.17 & 0.30 & 0.54 & 0.55 & 0.53 & 0.63 & 1.16 \\
\hline \multicolumn{11}{|c|}{ In an acoustically rigid shield } \\
\hline 10 & $f_{C}, \mathrm{~Hz}$ & 85.6 & 91.1 & 86.8 & 78.0 & 61.4 & 48.7 & 36.5 & 24.0 & 17.5 \\
\hline 11 & $f_{3}, \mathrm{~Hz}$ & 96.5 & 92.2 & 86.3 & 77.2 & 61.3 & 48.3 & 36.4 & 24.1 & 17.3 \\
\hline 12 & $\delta, \%$ & -12.75 & -1.22 & 0.63 & 0.98 & 0.19 & 0.90 & 0.22 & -0.38 & 1.02 \\
\hline
\end{tabular}

Also, a formula was derived to estimate the resonant frequency of the resonator when radiated into a half-space:

$$
f=\frac{c}{2 \pi} \sqrt{\frac{(V-\Delta V) \cdot\left(h+\frac{7}{3 \pi} r+\frac{4}{3 \cdot \arccos \left(\frac{r}{R}\right)} r\right)}{(V)}} .
$$


To check the proposed formulas (3) and (4), models of resonators with radiation into unlimited space and half-space were constructed for various resonant frequencies. The results of modeling and calculations are shown in Tables 2, 3.

Table 2

Resonator frequency tuned to $50 \mathrm{~Hz}$

\begin{tabular}{|c|c|c|c|c|c|c|c|c|c|c|}
\hline No. & Parameter & \multicolumn{9}{|c|}{ Parameter value } \\
\hline \multicolumn{11}{|c|}{ Without acoustic design } \\
\hline 1 & $f_{1}, \mathrm{~Hz}$ & & & & & 50 & & & & \\
\hline 2 & $r, \mathrm{~m}$ & & & & & 0.01 & & & & \\
\hline 3 & $h, \mathrm{~m}$ & 0.5 & 0.2 & 0.1 & 0.05 & 0.02 & 0.01 & 0.005 & 0.002 & 0.001 \\
\hline 4 & $R, \mathrm{~m}$ & 0.0564 & 0.0765 & 0.0963 & 0.1214 & 0.1647 & 0.2075 & 0.2615 & 0.3549 & 0.4471 \\
\hline 5 & $f_{C}, \mathrm{~Hz}$ & 47.6 & 48.0 & 46.7 & 44.1 & 37.9 & 31.5 & 24.8 & 17.0 & 12.4 \\
\hline 6 & $f_{2}, \mathrm{~Hz}$ & 49.1 & 48.0 & 46.2 & 43.2 & 36.8 & 30.4 & 23.8 & 16.2 & 11.8 \\
\hline 7 & $\delta, \%$ & -3.15 & 0.00 & 1.07 & 2.04 & 2.90 & 3.49 & 4.03 & 4.71 & 4.84 \\
\hline 8 & $f_{3}, \mathrm{~Hz}$ & 49.3 & 48.3 & 46.8 & 44.0 & 37.8 & 31.4 & 24.6 & 16.7 & 12.1 \\
\hline 9 & $\delta, \%$ & -3.47 & -0.70 & -0.25 & 0.12 & 0.32 & 0.43 & 0.88 & 1.76 & 2.34 \\
\hline \multicolumn{11}{|c|}{ In an acoustically rigid shield } \\
\hline 10 & $f_{C}, \mathrm{~Hz}$ & 47.5 & 47.9 & 46.4 & 43.6 & 37.2 & 30.9 & 24.3 & 16.6 & 12.1 \\
\hline 11 & $f_{3}, \mathrm{~Hz}$ & 49.11 & 48.02 & 46.32 & 43.40 & 37.14 & 30.90 & 24.32 & 16.63 & 12.11 \\
\hline 12 & $\delta, \%$ & -3.38 & -0.25 & 0.18 & 0.47 & 0.17 & 0.00 & -0.10 & -0.21 & -0.11 \\
\hline
\end{tabular}

Table 3

Resonator frequency tuned to $25 \mathrm{~Hz}$

\begin{tabular}{|c|c|c|c|c|c|c|c|c|c|c|}
\hline No. & Parameter & \multicolumn{9}{|c|}{ Parameter value } \\
\hline \multicolumn{11}{|c|}{ Without acoustic design } \\
\hline 1 & $f_{1}, \mathrm{~Hz}$ & & & & & 25 & & & & \\
\hline 2 & $r, \mathrm{~m}$ & & & & & 0.005 & & & & \\
\hline 3 & $h, \mathrm{~m}$ & 0.5 & 0.2 & 0.1 & 0.05 & 0.02 & 0.01 & 0.005 & 0.002 & 0.001 \\
\hline 4 & $R, \mathrm{~m}$ & 0.0564 & 0.0765 & 0.0963 & 0.1214 & 0.1647 & 0.2075 & 0.2615 & 0.3549 & 0.4471 \\
\hline 5 & $f_{C}, \mathrm{~Hz}$ & 24.6 & 24.5 & 24.1 & 23.2 & 21.2 & 18.7 & 15.6 & 11.2 & 8.3 \\
\hline 6 & $f_{2}, \mathrm{~Hz}$ & 24.8 & 24.5 & 24.0 & 23.1 & 20.9 & 18.4 & 15.2 & 10.9 & 8.1 \\
\hline 7 & $\delta, \%$ & -0.63 & 0.10 & 0.42 & 0.37 & 1.21 & 1.70 & 2.47 & 2.58 & 2.27 \\
\hline 8 & $f_{3}, \mathrm{~Hz}$ & 24.76 & 24.50 & 24.04 & 23.20 & 21.11 & 18.62 & 15.50 & 11.18 & 8.34 \\
\hline 9 & $\delta, \%$ & -0.66 & 0.01 & 0.24 & 0.01 & 0.41 & 0.41 & 0.65 & 0.16 & -0.47 \\
\hline \multicolumn{11}{|c|}{ In an acoustically rigid shield } \\
\hline 10 & $f_{C}, \mathrm{~Hz}$ & 24.6 & 24.5 & 24.1 & 23.4 & 21.4 & 18.9 & 15.7 & 11.3 & 8.4 \\
\hline 11 & $f_{3}, \mathrm{~Hz}$ & 24.78 & 24.53 & 24.10 & 23.31 & 21.32 & 18.90 & 15.82 & 11.49 & 8.60 \\
\hline 12 & $\delta, \%$ & -0.71 & -0.12 & -0.01 & 0.40 & 0.39 & -0.01 & -0.79 & -1.70 & -2.34 \\
\hline
\end{tabular}




\section{Discussion}

The proposed resonant frequency calculation method makes it possible to estimate the resonator resonant frequency more accurately. The relative inaccuracy of calculations according to the proposed formulas (3) and (4) is less than $1 \%$, given that $r / R<0.25$. The increasing inaccuracy with small $\mathrm{h}$ is caused by the model, since the accuracy of the resonant frequency in the model was $0.1 \mathrm{~Hz}$.

Similar investigations but for cylindrical cavity were described in [12]. The accuracy of determining the resonant frequency at $2 \%$ was obtained in this article. However, in the analytical expressions, there is a coefficient that depends on the size of the neck and is determined in the table and only for the length of the neck from $0.5 \mathrm{~mm}$ to $5 \mathrm{~mm}$, which doesn't allow applying the obtained expressions to other dimensions of the neck.

In the articles $[13,14]$, although the resonant frequencies weren't directly determined, but the losses of sound energy occurring in the resonators were studied. According to the graphs given in these articles, the discrepancy between the theoretically calculated loss maxima and the values measured in practice is not less than $1 \%$. That means that the accuracy of the results obtained in this article is comparable to the results of other researches.

The main restrictions on the use of the results here are:

1) $r / R<0.25$ (to achieve such a high accuracy of the results);

2) for the resonator in acoustically rigid shield it's a case of the infinite rigid shield. This means that the dimensions of the shield must be much larger than the dimensions of the resonator;

3) Only spherical Helmholtz resonators were investigated in this article, therefore these results can't be used for other shapes of resonators.

Similar investigations and analytical calculations should be carried out for the other shapes of resonators in the future such as cylindrical and rectangular, which is a very important task, according to the existence of the publications. Moreover, investigations [7] show that the shape of the neck also has a significant effect on the measurement results, which also requires careful study.

\section{Conclusions}

1. The presented formula (3) increases the accuracy of calculation the Helmholtz resonator resonant frequency in free field. The calculation error is no more than $1 \%$, given that $r / R<0.25$.

2 . Research has shown that the resonator acoustic design, specifically placed into an acoustically rigid shield, leads to its resonant frequency change.

3. Formula (4) provides sufficient resonator resonant frequency calculation results (cylindrical neck form and a spherical volume), which is located in an infinite size acoustically rigid shield.

4. Resonance frequency changes quite significantly when the acoustic design changes. This fact has to be taken into account during acoustic metamaterials construction.

\section{References}

[1] Mahesh, K., Mini, R. S. (2019). Helmholtz resonator based metamaterials for sound manipulation. Journal of Physics: Conference Series, 1355, 012031. doi: http://doi.org/10.1088/1742-6596/1355/1/012031

[2] Yang, X., Yin, J., Yu, G., Peng, L., Wang, N. (2015). Acoustic superlens using Helmholtz-resonator-based metamaterials. Applied Physics Letters, 107 (19), 193505. doi: http://doi.org/10.1063/1.4935589

[3] Feng, Q., Li, Q., Li-Wei, W., Xiao-Zhou, L., Xiu-Fen, G. (2016). Tunable acoustic radiation pattern assisted by effective impedance boundary. Chinese Physics B, 25 (2), 024301. doi: http://doi.org/10.1088/1674-1056/25/2/024301

[4] Yamamoto, T. (2018). Acoustic metamaterial plate embedded with Helmholtz resonators for extraordinary sound transmission loss. Journal of Applied Physics, 123 (21), 215110. doi: http://doi.org/10.1063/1.5025570

[5] Jiménez, N., Huang, W., Romero-García, V., Pagneux, V., Groby, J.-P. (2016). Ultra-thin metamaterial for perfect and quasi-omnidirectional sound absorption. Applied Physics Letters, 109 (12), 121902. doi: http://doi.org/10.1063/1.4962328

[6] Etaix, N., Crawford, K., Voisey, R., Hopper, H. (2016). Redesigning Helmholtz resonators to achieve attenuation at multiple frequencies. International Congress on Acoustics 2016. Buenos Aires. Available at: http://www.ica2016.org.ar/ica2016proceedings/ica2016/ICA2016-0491.pdf 
[7] Cai, C., Mak, C.-M., Shi, X. (2017). An extended neck versus a spiral neck of the Helmholtz resonator. Applied Acoustics, 115, 74-80. doi: http://doi.org/10.1016/j.apacoust.2016.08.020

[8] Han, M. (2008). Sound reduction by a Helmholtz resonator. Available at: https://preserve.lehigh.edu/cgi/viewcontent.cgi?article $=2015 \&$ context $=$ etd

[9] Abbad, A., Atalla, N., Ouisse, M., Doutres, O. (2019). Numerical and experimental investigations on the acoustic performances of membraned Helmholtz resonators embedded in a porous matrix. Journal of Sound and Vibration, 459, 114873. doi: http:// doi.org/10.1016/j.jsv.2019.114873

[10] Didkovskiy, V., Naida, S., Zaets, V. (2019). Experimental study into the Helmholtz resonators' resonance properties over a broad frequency band. Eastern-European Journal of Enterprise Technologies, 1 (5 (97)), 34-39. doi: http://doi.org/10.15587/17294061.2019.155417

[11] Engineering Acoustics/Noise control with self-tuning Helmholtz resonators. Available at: https://en.wikibooks.org/wiki/Engineering_Acoustics/Noise_control_with_self-tuning_Helmholtz_resonators

[12] Komkin, A. I., Mironov, M. A., Yudin, S. I. (2012). On the attached length of orifices. Acoustical Physics, 58 (6), $628-632$. doi: http://doi.org/10.1134/s1063771012050090

[13] Chanaud, R. C. (1994). Effects Of Geometry On The Resonance Frequency Of Helmholtz Resonators. Journal of Sound and Vibration, 178 (3), 337-348. doi: http://doi.org/10.1006/jsvi.1994.1490

[14] Chaitanya, P., Munjal, M. L. (2011). Effect of wall thickness on the end corrections of the extended inlet and outlet of a double-tuned expansion chamber. Applied Acoustics, 72 (1), 65-70. doi: http://doi.org/10.1016/j.apacoust.2010.09.001 